\title{
Fibration structure in toric hypersurface Calabi-Yau threefolds
}

\section{Yu-Chien Huang and Washington Taylor}

Center for Theoretical Physics, Department of Physics, Massachusetts Institute of Technology, 77 Massachusetts Avenue, Cambridge, MA 02139, U.S.A.

E-mail: yc_huang@mit.edu, wati@mit.edu

ABSTRACT: We find through a systematic analysis that all but 29,223 of the 473.8 million $4 \mathrm{D}$ reflexive polytopes found by Kreuzer and Skarke have a 2D reflexive subpolytope. Such a subpolytope is generally associated with the presence of an elliptic or genus one fibration in the corresponding birational equivalence class of Calabi-Yau threefolds. This extends the growing body of evidence that most Calabi-Yau threefolds have an elliptically fibered phase.

KeYwords: Differential and Algebraic Geometry, F-Theory, Superstring Vacua

ArXIV EPrint: 1907.09482 


\section{Contents}

1 Introduction 1

2 Reflexive subpolytopes and fibrations 2

2.1 Methodology 2

2.2 Implementation 4

2.3 Fibrations of polytopes versus toric varieties 4

3 Results $\quad 7$

$\begin{array}{lll}3.1 & \text { Fiber analysis of all reflexive 4D polytopes } & 7\end{array}$

$\begin{array}{lll}3.2 & \text { Asymptotics at small } h^{1,1} & 7\end{array}$

$\begin{array}{lll}3.3 & \text { Mirror symmetry } & 10\end{array}$

$\begin{array}{lll}4 & \text { Discussion } & 10\end{array}$

\section{Introduction}

Calabi-Yau manifolds have been studied intensively by physicists and mathematicians over the last several decades, since the first realization that these geometric spaces can be used to construct supersymmetric compactifications of string theory [1]. Despite much work, the question of whether the number of distinct topological types of Calabi-Yau threefolds is finite or infinite [2] is still unresolved. The special class of Calabi-Yau manifolds that have an elliptic or genus one fibration, on the other hand, is better understood mathematically and it has been proven that there are a finite number of distinct Calabi-Yau threefolds of this type, up to birational equivalence [3]. In recent years, elliptic Calabi-Yau manifolds have been studied in great detail by physicists due to their role in the nonperturbative formulation of string theory known as F-theory [4-6]. Using a combination of methods and insights from mathematics and physics, and building on Grassi's results for minimal models of the bases that support elliptic fibrations [7], we have a good global understanding of the connected space of elliptic Calabi-Yau threefolds, the bounds on their Hodge numbers, and a systematic approach to constructing such threefolds that can be used to essentially enumerate all elliptic Calabi-Yau threefolds at large $h^{2,1}$, though there are technical issues that make a complete enumeration at smaller values of $h^{2,1}$ currently out of reach (see e.g. [8-13]).

There is a growing body of evidence that most known Calabi-Yau threefolds are in fact elliptic or genus one fibered (at least up to birational equivalence). Some of the largest known sets of Calabi-Yau threefolds are the 7,890 complete intersection CalabiYau (CICY) threefolds [14], more generalized complete intersection Calabi-Yaus [15], and the Calabi-Yau threefolds constructed as hypersurfaces in toric varieties associated with 
the 473.8 million reflexive $4 \mathrm{D}$ polytopes [16]. It was shown in $[17,18]$ that $99.3 \%$ of the CICY threefolds have an "obvious" genus one or elliptic fibration, and that many of these threefolds admit many such fibrations; similar results hold for discrete quotients of the CICY threefolds [19]. It was shown in [20] that many polytopes in the Kreuzer-Skarke (KS) database [21] have a structure compatible with a K3 fibration. A systematic construction of elliptic CY threefolds at large Hodge numbers over toric base surfaces [22] showed that all Hodge number pairs in the KS database with $h^{1,1} \geq 240$ or $h^{2,1} \geq 240$ are associated with such elliptic Calabi-Yau threefolds. A systematic direct study of the fibration structure of the polytopes in the KS database was initiated in [23]; in that paper we found that all polytopes associated with Calabi-Yau threefolds having $h^{1,1} \geq 150$ or $h^{2,1} \geq 150$ have a reflexive 2D subpolytope, indicating a structure compatible with the presence of an elliptic or genus one fibration for the associated CY threefolds. In that paper we also found empirically that at small $h^{1,1}$, the fraction of $4 \mathrm{D}$ polytopes lacking such a reflexive 2D subpolytope drops roughly as $2^{-h^{1,1}}$, and we gave some analytic arguments for why these results may naturally be expected.

In this paper we complete the program begun in [23], and we report on the results of a complete analysis of all 473.8 million reflexive $4 \mathrm{D}$ polytopes for reflexive $2 \mathrm{D}$ subpolytopes. The upshot of the analysis is that the Calabi-Yau threefolds that correspond to the toric hypersurface construction seem to be dominated by those that are elliptic or genus one fibered in some phase, so that in fact most known Calabi-Yau threefolds are birationally equivalent to one with an elliptic or genus one fibration. Section 2 contains a description of the methodology used and a brief discussion of how the existence of a reflexive 2D subpolytope is related to the existence of an elliptic or genus one fibration of the associated Calabi-Yau threefolds. Section 3 describes the results of the subpolytope analysis, and section 4 contains a summary of the results and discussion.

\section{Reflexive subpolytopes and fibrations}

The basic question we consider here is which reflexive 4D polytopes have $2 \mathrm{D}$ subpolytopes that pass through the origin and hence act as fibers. From the work of Batyrev [24], we know that a hypersurface in a toric variety associated with a reflexive $4 \mathrm{D}$ polytope generically gives a smooth Calabi-Yau threefold; thus, a reflexive 2D subpolytope $\nabla_{2}$ of a reflexive $4 \mathrm{D}$ polytope $\nabla$ suggests the presence of a genus one or elliptic fibration for a corresponding Calabi-Yau threefold [25].

The problem of identifying 2D subpolytopes from the combinatorial data of a $4 \mathrm{D}$ polytope is described and discussed in some detail in [23, 26, 27]. We use here the notation and conventions of $[22,23]$, to which the reader is referred for further background and references.

\subsection{Methodology}

The algorithm that we have used to identify the existence of a $2 \mathrm{D}$ reflexive subpolytope is a streamlined version of the algorithms discussed in [23, 26, 27]. The basic idea is to 
determine whether any pair of rays in $\nabla \cap \mathbb{Z}^{4}$ generate a $2 \mathrm{D}$ sublattice of $\mathbb{Z}^{4}$ that intersects $\nabla$ in a set of points that form a $2 \mathrm{D}$ polytope containing the origin as an interior point.

We use as starting data the set of lattice points $L=\nabla \cap \mathbb{Z}^{4} \subset \nabla$ and the set of vertices $V$ of the dual polytope $\Delta=\nabla^{*}=\{w:\langle w, p\rangle \geq-1, \forall p \in \nabla\}$. For each lattice point $p \in L$, we compute

$$
s(p)=\max _{v \in V}\langle p, v\rangle .
$$

We then make a list of all the points in $L$ with $s(p) \in\{1,2,3\}$

$$
S_{i}=\{p \in L: s(p)=i\} .
$$

The purpose of this set of lists is to reduce the number of computations needed to check for the presence of the $2 \mathrm{D}$ subpolytope; the data in $S_{1}, S_{2}, S_{3}$ is all that is needed to check whether such a subpolytope exists. This reduces the computational cost of the analysis substantially when the number of points in $L$ is large.

There are 16 distinct $2 \mathrm{D}$ reflexive polytopes, discussed in the physics context in [2730]. For each of these reflexive polytopes, it is straightforward to check whether one of the following conditions holds:

$$
\begin{aligned}
& \text { I. } \exists x, y \in S_{1}: x \neq \pm y \text { and }-x,-y \in S_{1} \\
& \text { II. } \exists x, y \in S_{2}: x \neq y \text { and }\left(-(x+y) \in S_{1} \text { or }-(x+y) \in S_{2}\right) \\
& \text { III. } \exists x, y \in S_{3}: x \neq y \text { and }-(x+y) / 2 \in S_{1} .
\end{aligned}
$$

In particular, with the standard ordering of the 16 fiber polytopes (used in e.g. [27] and [23]), condition I holds for fibers $2,5,7,8,9,11,12,13,14,15,16$; of the fibers where condition I fails, condition II holds for fibers 1, 3, 6, 10; and condition III holds for the sole remaining case, fiber 4. By simply checking these conditions for the $S_{i}$ for each polytope, we can ascertain whether or not there is a reflexive $2 \mathrm{D}$ subpolytope $\nabla_{2}$ of $\nabla$. The fact that it is necessary for one of these conditions to hold for there to exist a reflexive 2D subpolytope follows from the explicit enumeration of cases given above. The fact that it is sufficient for these conditions to hold follows from the fact that each of the three conditions guarantees the presence of a set of lattice points in $L$ that form a convex 2 D polytope containing the origin in the interior; in particular, if we define a basis so that $x, y$ are the vectors $(1,0)$ and $(0,1)$ then in addition to $x, y$ condition I implies that $(-1,0)$ and $(0,-1)$ are in $L$ and condition II implies that $(-1,-1)$ is in $L$, while condition III implies that there is a basis where $x=(1,1), y=(1,-1)$ and $(-1,0)$ are in $L$. Since the origin is the only point in the interior of $\nabla$, it must also be the only lattice point in the interior of $L$, so the convex hull of the set of points in $L$ must form a 2D reflexive subpolytope.

Note that once the existence of a $2 \mathrm{D}$ subpolytope $\nabla_{2}$ is determined, it is straightforward to identify the full set of lattice points from $\nabla$ that are in $\nabla_{2}$ as those that lie in the plane spanned by the pair of points $x, y$ that satisfy one of the above 3 conditions. The specific fiber type of $\nabla_{2}$ is then uniquely determined by the numbers of points $n_{i}=\left|\nabla_{2} \cap S_{i}\right|$ for $i=1, \ldots, 5$. For a determination of fiber types it is therefore helpful to initially determine the sets $(2.2)$ for $i=1, \ldots, 5$, and then we only need to compute which of these lattice 


\begin{tabular}{|c|c|}
\hline Fiber number & $\left(n_{1}, \ldots, n_{5}\right)$ \\
\hline 1 & $(0,3,0,0,0)$ \\
2 & $(4,0,0,0,0)$ \\
3 & $(2,2,0,0,0)$ \\
4 & $(2,0,2,0,0)$ \\
5 & $(4,1,0,0,0)$ \\
6 & $(2,2,1,0,0)$ \\
7 & $(6,0,0,0,0)$ \\
8 & $(4,1,1,0,0)$ \\
9 & $(4,2,0,0,0)$ \\
10 & $(2,2,1,0,1)$ \\
11 & $(4,2,1,0,0)$ \\
12 & $(6,1,0,0,0)$ \\
13 & $(4,2,2,0,0)$ \\
14 & $(6,2,0,0,0)$ \\
15 & $(8,0,0,0,0)$ \\
16 & $(6,3,0,0,0)$ \\
\hline
\end{tabular}

Table 1. Distinguishing values of $n_{i}=\left|\nabla_{2} \cap S_{i}\right|$ for the 16 fibers.

points lie in the plane spanned by $x, y$. The values of $n_{i}$ associated with each of the 16 fiber types are listed in table 1. This information is also contained in appendix A of [31]; the numbers associated with the lattice points in each reflexive $2 \mathrm{D}$ polytope there are $n_{i}+1$ in this notation. While it is thus straightforward in any given case to determine the fiber type, we have not collected this information in the full database study carried out here, and in most of this paper we focus simply on the binary question for each $4 \mathrm{D}$ reflexive polytope of whether there is a $2 \mathrm{D}$ reflexive subpolytope fiber. A more detailed analysis of the fiber types at large and small Hodge numbers was carried out in [23].

\subsection{Implementation}

To carry out the calculation over all 473.8 million reflexive $4 \mathrm{D}$ polytopes, we first organized the information in the KS database [21] in files indexed by the Hodge numbers of the associated Calabi-Yau threefolds. We then used the palp software package to compute the set of lattice points $L \subset \nabla$ and dual vertices $V \subset \Delta$ for each polytope. The core algorithm described in the previous subsection to identify $2 \mathrm{D}$ reflexive subpolytopes was implemented in the language Julia, which combines ease of programming with efficiency through justin-time compiling. Checking all 473.8 million cases took roughly 4 days on a single core of a Linux laptop. The Julia code is available at [32].

\subsection{Fibrations of polytopes versus toric varieties}

While identifying a reflexive $2 \mathrm{D}$ subpolytope of the $4 \mathrm{D}$ polytope $\nabla$ suggests the presence of an elliptic or genus one fibration for the associated toric varieties, there are some subtleties in making this connection precise. ${ }^{1}$ While some analyses of fibration structures have focused

\footnotetext{
${ }^{1}$ Thanks to Antonella Grassi for emphasizing the importance of these issues.
} 
on the structure of the polytope alone - sometimes in the language of "tops" $[28,33]$ as mentioned in [25] and discussed in more detail in [26] not every triangulation of a 4D polytope $\nabla$ with a $2 \mathrm{D}$ reflexive subpolytope $\nabla_{2}$ leads to a toric morphism that maps the subpolytope $\nabla_{2}$ to the origin of the toric fan for the natural corresponding $2 \mathrm{D}$ base. A condition stated in [25] is that the base $B$ of such a fibration should be identified by constructing the $2 \mathrm{D}$ toric variety from the set of primitive rays in the image of $\nabla$ under the projection that takes $\nabla_{2} \rightarrow 0$. Indeed, in many cases, such as the "standard stacking" polytopes corresponding to many generic and (Tate) tuned Weierstrass models over a given base, one can use the structure of the polytopes and tops to determine the base and additional tuned Kodaira singularity types to directly construct a Weierstrass model for an elliptic fibration over the given base, thus identifying a Calabi-Yau threefold that is elliptically fibered and has the requisite Hodge numbers associated with the polytope, circumventing the explicit construction of fans compatible with a toric morphism from $\nabla$ to $\nabla_{2}$ [22]. Particularly for more complicated fibrations with general fibers and twists, however, there is no systematic methodology for implementing such a direct construction; and in any case, it is desirable to know in general which triangulations of $\nabla$ are compatible with the fibration structure, and whether in fact such triangulations always exist.

Some of these questions regarding triangulations will be addressed in detail elsewhere [34, 35]; here we simply summarize a few key points. First, for a given polytope, in addition to triangulations compatible with a toric morphism to the base associated with the full set of rays in the image of the projection, there can also be triangulations compatible only with a toric morphism to a base with smaller $h^{1,1}$, which are associated with non-flat elliptic fibrations. ${ }^{2}$ An example is given by the "standard stacking" polytope associated with the generic elliptic fibration over the base $\mathbb{F}_{1}$ with the fiber $\mathbb{P}^{2,3,1}$. The vertices of this polytope are $(0,0 ; 1,0),(0,0 ; 0,1),\left(u_{i}^{(B)} ;-2,-3\right)$, where $u_{i}^{(B)}=(0,1),(1,0),(0,-1),(-1,-1)$ are the vertices of the polytope associated with the base $\mathbb{F}_{1}$. The resulting polytope $\nabla$ has triangulations associated with two different Calabi-Yau geometries related by a flop. The part of these triangulations associated with the $2 \mathrm{D}$ face $B \cong \mathbb{F}_{1}$ of $\nabla$ is shown in figure 1. The first triangulation leads to a toric morphism associated with an elliptic fibration over $\mathbb{F}_{1}$. The second triangulation, on the other hand, corresponds to a non-flat toric morphism giving a non-flat elliptic fibration in which the divisor associated with $v_{3}$ is contracted to a point in the toric base $\mathbb{P}^{2}$. For bases with larger $2 \mathrm{D}$ polytopes, associated with Calabi-Yau threefolds with larger $h^{1,1}$, we expect many more non-flat elliptic and genus one fibrations of this type to arise from triangulations of this kind. In other cases, triangulations are associated with toric morphisms to a singular base, where some rays are not included in the base. Furthermore, there can be cases where a triangulation is such that there is no toric morphism to any base associated with the projection of $\nabla_{2}$ to the origin, and there are cases where there is no triangulation at all satisfying the usual (fine, regular, star) conditions that is compatible with such a toric morphism. Nonetheless, we can demonstrate that whenever there is a reflexive subpolytope $\nabla_{2} \subset \nabla$ there is always a

\footnotetext{
${ }^{2}$ Non-flat elliptic fibrations were encountered and described in this toric context in [26, 27]; non-flat fibrations were and analyzed in more general physics and F-theory contexts in [17, 22, 36-44].
} 


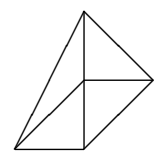

(a)

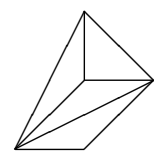

(b)

Figure 1. Two triangulations of the 2D face corresponding to the base resulting from distinct Calabi-Yau phase triangulations of the 4D polytope associated with the generic elliptic fibration over the Hirzebruch surface $\mathbb{F}_{1}$; the first triangulation gives a toric variety with a toric morphism to the base $\mathbb{F}_{1}$, while the second triangulation gives a variety with a non-flat toric morphism to $\mathbb{P}^{2}$.

triangulation of $\nabla$ giving a toric variety with a fan $\Sigma$ compatible with a toric morphism $\pi: \Sigma \rightarrow \Sigma_{B_{s}}$, where $B_{s}$ is the (generally singular) toric base defined by taking only the primitive rays associated with the projection of certain vertices of the polytope $\nabla$ under the map $\pi: \nabla_{2} \rightarrow 0$ (in many cases in practice the base can be taken to be a refinement $B^{\prime}$ of $B_{s}$ ). This triangulation can be constructed by starting with a triangulation of the $4 \mathrm{D}$ polytope defined by $\nabla_{2}$ and a set of points associated with (necessarily primitive) vertices $u_{i}$ of $\nabla$ that project to the vertices of $\pi(\nabla)$, using the natural orderings of rays in $\nabla_{2}$ and $\pi(\nabla)$. The resulting toric variety is generally highly singular, and the fan can then be refined by including the remaining lattice points of $\nabla$ in an arbitrary refinement of the original singular triangulation; this final triangulation respects the toric morphism to $B_{s}$ (or a refinement $B^{\prime}$ thereof), and gives a genus one or elliptic fibration of the resulting toric variety. The fan constructed in this way, however, generally does not give a triangulation of the full polytope $\nabla$ that satisfies the star condition (though it is a star triangulation of a non-convex polytope contained within $\nabla$ ), so that the resulting Calabi-Yau phase does not directly fit into the Batyrev framework and requires the more general structure of "vex" polytopes for toric varieties that are not weak Fano developed in [45]. The need to include ambient toric varieties where the anticanonical class has a base locus is related to the presence of "non-Higgsable clusters" [46], associated with rigid subvarieties of the Calabi-Yau hypersurface that are present everywhere in the moduli space of that phase. The presence of a triangulation of this type guarantees a Calabi-Yau phase with an elliptic or genus one fibration, although particularly in higher dimensions there can be singularities without a Calabi-Yau resolution [47, 48]. The issue of regularity, corresponding to positivity in the Kähler cone, must also be addressed. We leave a more detailed explication of these issues to future work; the upshot of this discursion is that the presence of a reflexive 2D subpolytope $\nabla_{2} \subset \nabla$ is generally associated with at least one triangulation giving an elliptic or genus one fibration of a corresponding toric variety.

We should also emphasize that while the presence of a reflexive 2D subpolytope seems in general to be sufficient for a genus one or elliptic fiber of an associated Calabi-Yau threefold, this condition is not necessary. In studying subpolytopes of $\nabla$, we are only finding the "obvious" fibers that are encoded in a natural way in the toric structure. There can also be fibrations that are not represented torically, which could be analyzed by a more complete treatment of the structure of the Calabi-Yau threefold using the full set of triple intersection numbers and Kähler cone information, as was done for CICY threefolds in [18]. 


\section{Results}

\subsection{Fiber analysis of all reflexive $4 \mathrm{D}$ polytopes}

Extending the work initiated in [23], we have checked all 473.8 million reflexive $4 \mathrm{D}$ polytopes in the Kreuzer-Skarke database [21] for the presence of at least one reflexive 2D subpolytope passing through the origin. We find that all but 29,223 (i.e. 99.994\%) of the $4 \mathrm{D}$ reflexive polytopes have such a fiber.

The 1,395 distinct Hodge numbers of the 29,223 cases without reflexive 2D subpolytopes are shown in red in figure 2. The largest $h^{1,1}$ for a Calabi-Yau threefold associated with a non-fibered $4 \mathrm{D}$ polytope comes from the case with Hodge numbers $(140,62)$, as previously identified in [23]. The next-largest values of $h^{1,1}$ come from polytopes associated with Calabi-Yau threefolds having Hodge numbers

$$
(114,60),(113,61) \times 2,(112,62),(111,63) \times 2,(108,62) \times 2,(99,63) \times 2, \ldots
$$

It is very interesting that the polytopes corresponding with large $h^{1,1}$ that do not have 2D reflexive subpolytopes have Hodge numbers $h^{2,1}$ in the narrow range $60-63$; the first exception as $h^{1,1}$ decreases has the Hodge numbers $(95,55)$, and for all $h^{1,1}>61$ the nonfibered cases have $h^{2,1}$ in the range $48-65$. It would be interesting to understand better whether this family of polytopes has some common structure associated with the lack of an elliptic or genus one fibration for the corresponding toric varieties. Indeed, there are similarities between some of these cases: in particular, for example, the polytope giving Hodge numbers $(114,60)$ has only a slight difference in one vertex from one of the polytopes giving Hodge numbers $(113,61)$; we leave a more detailed analysis of common structure in these polytopes for further work, however.

The distribution of polytopes without a 2D reflexive subpolytope is graphed in figure 3 , and compared to the total number of polytopes. While the total number of reflexive 4D polytopes at a given value of $h^{1,1}$ peaks at $h^{1,1}=27$ (with 16.7 million reflexive 4D polytopes at this value of $\left.h^{1,1}\right)$, the number without a reflexive $2 \mathrm{D}$ subpolytope peaks at $h^{1,1}=13$ (with 1,767 cases).

We have made available the results of our analysis at [32], where the list of 29,223 4D reflexive polytopes without a $2 \mathrm{D}$ reflexive subpolytope can be found in the format of [21].

\subsection{Asymptotics at small $h^{1,1}$}

In [23], a heuristic argument was given for why the fraction of Calabi-Yau threefolds without an elliptic or genus one fiber may decrease exponentially, and it was noted that at small values of $h^{1,1}$ the empirically computed value of this fraction from the KS database decreases roughly as $2^{-h^{1,1}}$. The heuristic argument is based on the assumption that the triple intersection numbers of the Calabi-Yau are essentially random and that the Kähler cone is a generalized quadrant defined by conditions $x_{i} \geq 0$ on the coefficients of the Kähler class in a natural basis of effective divisors. With the full set of data, we see that while this is not a bad approximation at small $h^{1,1}$, the tail of the distribution is considerably fatter; indeed, as pointed out in [23], the distribution must drop off less rapidly than $2^{-h^{1,1}}$ for the 


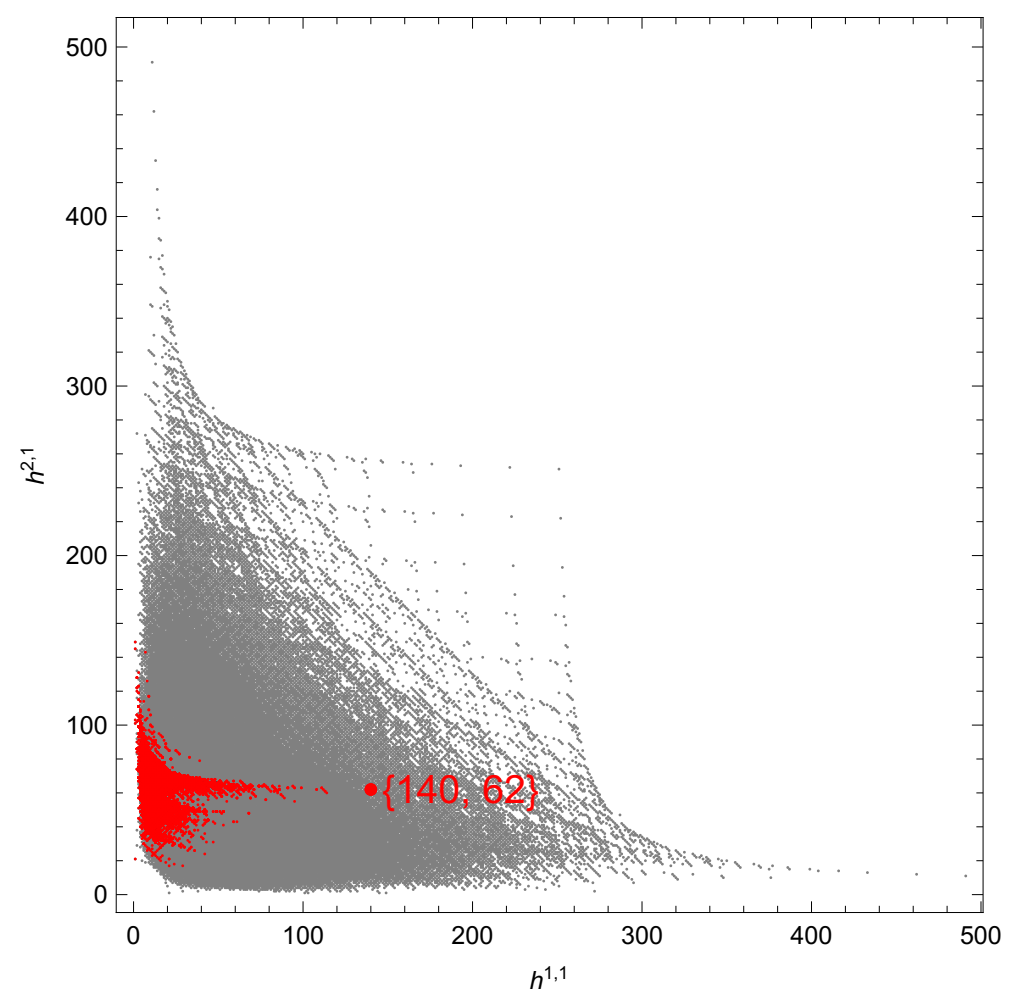

Figure 2. The Hodge numbers of the Calabi-Yau threefolds associated with $4 \mathrm{D}$ reflexive polytopes lacking a 2D reflexive subpolytope (red), plotted over the Hodge numbers of the full set of toric hypersurface Calabi-Yau threefolds (gray).

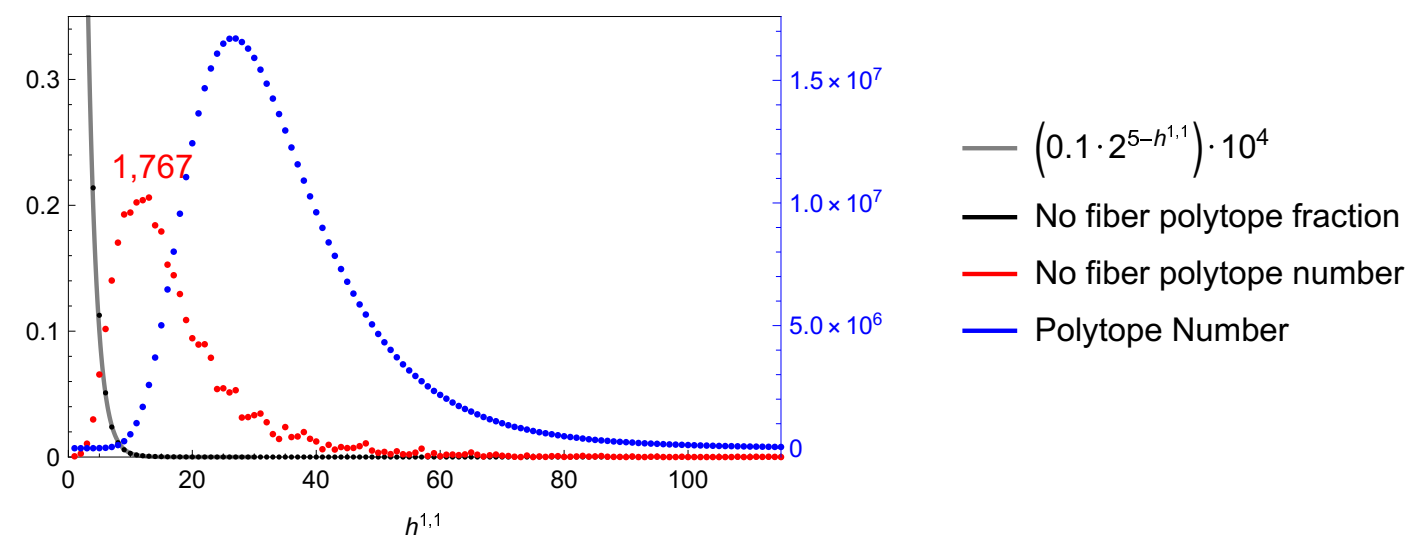

Figure 3. The distribution of $4 \mathrm{D}$ reflexive polytopes without a 2D reflexive subpolytope fiber (red) compared to the total number of $4 \mathrm{D}$ reflexive polytopes (blue) as a function of Hodge number $h^{1,1}$, and the fraction without fibers (gray). Note that the vertical axis is normalized differently for each of the three curves. 


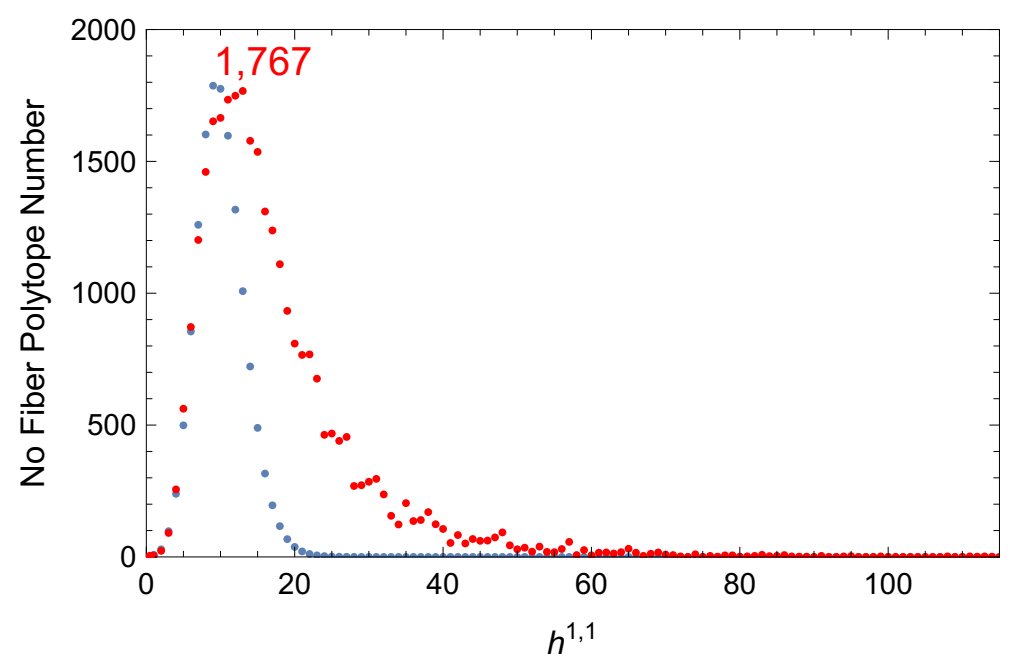

Figure 4. Illustration of the "fat tail" in the number of $4 \mathrm{D}$ reflexive polytopes without $2 \mathrm{D}$ reflexive toric fibers (red), compared to exponentially suppressed prediction (gray) based on heuristic analysis of elliptic fibrations for Calabi-Yau threefolds from [23].

large $h^{1,1}$ examples such as $(140,62)$ to lack fibers. The tail of the observed distribution is compared to the prediction based on the asymptotic estimate $c \cdot 2^{-h^{1,1}}$ fit to the small values of $h^{1,1}$ in figure 4 .

It is an interesting question for further work to determine why the observed distribution drops off less quickly than the exponential estimate. One possibility is that the toric structure is simply missing the elliptic/genus one fibration structure in many of the cases with larger values of $h^{1,1}$. While the presence of a reflexive 2D subpolytope is an indicator for an elliptic or genus one fibration for at least one phase of the associated Calabi-Yau threefold, such a subpolytope is not a necessary condition for a fibration. A fibration of an associated Calabi-Yau threefold may occur in a way that is simply not captured by the toric geometry of the ambient space into which the Calabi-Yau is embedded as a hypersurface. A similar situation was encountered in the analysis of the CICY CalabiYau threefolds in [18]; while many of the CICYs have "obvious" fibrations that can be seen in the structure of the defining matrices, there are also non-obvious fibrations that can be identified by a more careful analysis of the triple intersection numbers and Kähler cone of the Calabi-Yau. One might similarly expect that many non-toric fibrations may be present for the cases for which we have not identified a reflexive 2D subpolytope; this would be interesting to investigate further. Another possibility is that there is an error coming from the assumption that the Kähler cone can be approximated as a generalized quadrant. Indeed, a recent analysis [49] suggests that at large $h^{1,1}$ the Kähler cone becomes progressively more narrow. This may lead to a situation that makes it easier to avoid having an elliptic or genus one fibration as $h^{1,1}$ increases than the naive argument leading to the exponential decrease would suggest. Nonetheless, even though the tail of the distribution is a bit fatter than the naive exponential argument suggested, the drop-off is still relatively quick and the total number of reflexive $4 \mathrm{D}$ polytopes without a reflexive $2 \mathrm{D}$ subpolytope is really quite small. 


\subsection{Mirror symmetry}

One interesting application of this analysis may be to mirror symmetry. It was pointed out in [31] that in many cases where a polytope and its dual both admit a reflexive $2 \mathrm{D}$ subpolytope the mirror symmetry between the associated Calabi-Yau threefolds essentially factorizes between the fiber and the base. Since we have found fibers for most reflexive 4D polytopes, it suggests that this factorization may be relevant for most Calabi-Yau threefolds.

One interesting class of cases for further study are those where one side of a mirror pair is elliptic or genus one fibered and the other is not. For example, the mirror quintic with Hodge numbers $(101,1)$ has a fibration, while the quintic (like all Calabi-Yau threefolds with $h^{1,1}=1$ ) cannot have an elliptic or genus one fiber. One might hope to connect non-fibered threefolds like the quintic with the large connected set of elliptic Calabi-Yau threefolds through a transition, which may be related to how the mirror quintic is connected to other elliptic Calabi-Yau threefolds.

It is also interesting to consider cases where neither the polytope nor its mirror admit a manifest toric fibration. A systematic check shows that there are only 24 such polytopes $\nabla$ in the KS database that do not have a $2 \mathrm{D}$ reflexive subpolytope either for $\nabla$ or its dual $\Delta$. These 24 polytopes have the Hodge numbers

$$
(43,59),(44,44),(63,67),(64,66),(65,65) \text {. }
$$

It would be interesting to understand better whether there is some common structure underlying these polytopes and their associated Calabi-Yau threefolds, and whether they completely lack elliptic/genus one fibers or such fibers are simply not visible torically.

\section{Discussion}

We have carried out a systematic analysis of the full database of 473.8 million reflexive 4D polytopes and found that fewer than 30,000 of these lack a 2D reflexive subpolytope. It seems that the $99.994 \%$ of polytopes with a 2D reflexive fiber correspond to Calabi-Yau threefolds that are birationally equivalent to a Calabi-Yau threefold phase that has a genus one or elliptic fibration, though some technical aspects of the triangulations associated with the fibered phase of some polytopes remain to be resolved. This gives strong evidence supporting the hypothesis that most Calabi-Yau threefolds are elliptic or genus one fibered, up to birational equivalence; this in turn, if true, would indicate that the number of birational equivalence classes of Calabi-Yau threefolds is finite.

The apparent predominance of elliptic and genus one fibrations among Calabi-Yau threefolds has a number of interesting implications for physics. In particular, this has implications for the role of F-theory in understanding the global set of string compactifications. For 6D supergravity theories, F-theory seems to give a good global description of the space of possible string compactifications, and essentially all known string compactifications to $\mathcal{N}=16 \mathrm{D}$ supergravity theories have a dual description in F-theory. This is not true in $4 \mathrm{D}$, however; for example, heterotic compactification on a Calabi-Yau threefold only has a known F-theory dual description when the Calabi-Yau threefold is elliptic or 
genus one fibered (see e.g. [50]). If, however, indeed most Calabi-Yau threefolds have such a fibration, then most heterotic compactifications have an F-theory dual description. Thus, the results of this paper contribute evidence to the hypothesis that F-theory gives a very general representative sample of $\mathcal{N}=14 \mathrm{D}$ string theory vacua.

The analysis here of toric hypersurface Calabi-Yau threefolds and the analysis in [17, 18] of CICY threefolds give similar results for the overwhelming dominance of elliptic and genus one fibered Calabi-Yau threefolds for these large families. It would be interesting to extend this kind of analysis to other known Calabi-Yau threefolds that do not fit in these two families. A variety of other constructions can give other Calabi-Yau threefolds, particularly at small Hodge numbers (see [51] for a review), and it would be interesting to analyze the fibration structure of these other constructions. It would also be a good check on these results to consider the fibration structure of more general complete intersections in toric varieties; the fibers that may arise in such constructions were systematically analyzed in [52].

The analysis of this paper may be helpful in various systematic studies of CalabiYau threefolds and their properties that are relevant for physics, since there are many questions that are easier to answer for elliptic and genus one fibered Calabi-Yau threefolds than for general Calabi-Yau threefolds (see e.g. [53]). It would also be interesting to understand better the relatively small class of $4 \mathrm{D}$ reflexive polytopes without a reflexive 2D subpolytope; these correspond to Calabi-Yau threefolds without an (obvious) elliptic or genus one fiber, which seem in light of this analysis to form a rather special subset of the set of known Calabi-Yau threefolds.

One can ask similar questions about elliptic and genus one fibration structure for Calabi-Yau fourfolds. In [54] it was shown that the fraction of CICY Calabi-Yau fourfolds that has an obvious elliptic or genus one fibration (99.95\%) is even larger than the fraction of CICY Calabi-Yau threefolds with this property (99.3\%). There is no complete analysis of reflexive $5 \mathrm{D}$ polytopes, though there are some partial results in this direction [55] and some analysis of the fibration structure of these polytopes was carried out in [26]. In fact, direct construction of toric threefold bases that support elliptic and genus one toric hypersurface Calabi-Yau fourfolds shows that the number of such bases alone is extraordinarily large, on the order of $10^{3000}[48,56,57]$. Thus, the number of elliptic Calabi-Yau fourfolds that can be directly constructed in this way overwhelmingly exceeds any other class of known constructions for Calabi-Yau fourfolds. It is thus the case that the vast majority of known constructions of Calabi-Yau fourfolds are automatically elliptic or genus one fibered.

\section{Acknowledgments}

We would like to thank Lara Anderson, Anuj Apte, Per Berglund, Antonella Grassi, James Gray, Jim Halverson, Cody Long, Liam McAllister, Brent Nelson, Paul Oehlmann, Sakura Schafer-Nameki, Yi-Nan Wang and S.T. Yau for helpful discussions. This material is based upon work supported by the U.S. Department of Energy, Office of Science, Office of High Energy Physics under grant Contract Number DE-SC00012567. WT would like to thank the high-energy theory physics groups at UCSD and UCSC for hospitality during the time when most of this work was carried out. 
Open Access. This article is distributed under the terms of the Creative Commons Attribution License (CC-BY 4.0), which permits any use, distribution and reproduction in any medium, provided the original author(s) and source are credited.

\section{References}

[1] P. Candelas, G.T. Horowitz, A. Strominger and E. Witten, Vacuum configurations for superstrings, Nucl. Phys. B 258 (1985) 46 [INSPIRE].

[2] S.T. Yau, 'Open problems in geometry, Proc. Symp. Pure Math 54 (1993) 1.

[3] M. Gross, A finiteness theorem for elliptic Calabi-Yau threefolds, Duke Math. J. 74 (1994) 271.

[4] C. Vafa, Evidence for F-theory, Nucl. Phys. B 469 (1996) 403 [hep-th/9602022] [InSPIRE].

[5] D.R. Morrison and C. Vafa, Compactifications of F-theory on Calabi-Yau threefolds - I, Nucl. Phys. B 473 (1996) 74 [hep-th/9602114] [INSPIRE].

[6] D.R. Morrison and C. Vafa, Compactifications of F-theory on Calabi-Yau threefolds - II, Nucl. Phys. B 476 (1996) 437 [hep-th/9603161] [INSPIRE].

[7] A. Grassi, On minimal models of elliptic threefolds, Math. Ann. 290 (1991) 287.

[8] V. Kumar, D.R. Morrison and W. Taylor, Global aspects of the space of $6 D N=1$ supergravities, JHEP 11 (2010) 118 [arXiv: 1008.1062] [INSPIRE].

[9] D.R. Morrison and W. Taylor, Toric bases for 6D F-theory models, Fortsch. Phys. 60 (2012) 1187 [arXiv: 1204.0283] [INSPIRE].

[10] W. Taylor, On the Hodge structure of elliptically fibered Calabi-Yau threefolds, JHEP 08 (2012) 032 [arXiv: 1205.0952] [INSPIRE].

[11] W. Taylor and Y.-N. Wang, Non-toric bases for elliptic Calabi-Yau threefolds and $6 D$ F-theory vacua, Adv. Theor. Math. Phys. 21 (2017) 1063 [arXiv:1504.07689] [INSPIRE].

[12] S.B. Johnson and W. Taylor, Calabi-Yau threefolds with large $h^{2,1}$, JHEP 10 (2014) 023 [arXiv: 1406.0514] [INSPIRE].

[13] S.B. Johnson and W. Taylor, Enhanced gauge symmetry in $6 D$ F-theory models and tuned elliptic Calabi-Yau threefolds, Fortsch. Phys. 64 (2016) 581 [arXiv:1605.08052] [INSPIRE].

[14] P. Candelas, A.M. Dale, C.A. Lütken and R. Schimmrigk, Complete intersection Calabi-Yau manifolds, Nucl. Phys. B 298 (1988) 493 [INSPIRE].

[15] L.B. Anderson et al., A new construction of Calabi-Yau manifolds: generalized CICYs, Nucl. Phys. B 906 (2016) 441 [arXiv: 1507.03235] [InSPIRE].

[16] M. Kreuzer and H. Skarke, Complete classification of reflexive polyhedra in four-dimensions, Adv. Theor. Math. Phys. 4 (2002) 1209 [hep-th/0002240] [INSPIRE].

[17] L.B. Anderson, X. Gao, J. Gray and S.-J. Lee, Multiple fibrations in Calabi-Yau geometry and string dualities, JHEP 10 (2016) 105 [arXiv:1608.07555] [INSPIRE].

[18] L.B. Anderson, X. Gao, J. Gray and S.-J. Lee, Fibrations in CICY threefolds, JHEP 10 (2017) 077 [arXiv : 1708. 07907] [INSPIRE].

[19] L.B. Anderson, J. Gray and B. Hammack, Fibrations in non-simply connected Calabi-Yau quotients, JHEP 08 (2018) 128 [arXiv: 1805.05497] [INSPIRE]. 
[20] P. Candelas, A. Constantin and H. Skarke, An abundance of K3 fibrations from polyhedra with interchangeable parts, Commun. Math. Phys. 324 (2013) 937 [arXiv:1207.4792] [INSPIRE].

[21] M. Kreuzer and H. Skarke, http://hep.itp.tuwien.ac.at/ kreuzer/CY.html.

[22] Y.-C. Huang and W. Taylor, Comparing elliptic and toric hypersurface Calabi-Yau threefolds at large Hodge numbers, JHEP 02 (2019) 087 [arXiv: 1805.05907] [INSPIRE].

[23] Y.-C. Huang and W. Taylor, On the prevalence of elliptic and genus one fibrations among toric hypersurface Calabi-Yau threefolds, JHEP 03 (2019) 014 [arXiv: 1809.05160] [INSPIRE].

[24] V. Batyrev, Variations of the mixed Hodge structure of affine hypersurfaces in algebraic tori, Duke Math. J. 69 (1993) 349.

[25] M. Kreuzer and H. Skarke, Calabi-Yau four folds and toric fibrations, J. Geom. Phys. 26 (1998) 272 [hep-th/9701175] [inSPIRE].

[26] F. Rohsiepe, Fibration structures in toric Calabi-Yau fourfolds, hep-th/0502138 [INSPIRE].

[27] V. Braun, Toric elliptic fibrations and F-theory compactifications, JHEP 01 (2013) 016 [arXiv: 1110.4883] [INSPIRE].

[28] V. Bouchard and H. Skarke, Affine Kac-Moody algebras, CHL strings and the classification of tops, Adv. Theor. Math. Phys. 7 (2003) 205 [hep-th/0303218] [INSPIRE].

[29] V. Braun, T.W. Grimm and J. Keitel, Geometric engineering in toric F-theory and GUTs with U(1) gauge factors, JHEP 12 (2013) 069 [arXiv:1306.0577] [INSPIRE].

[30] D. Klevers et al., F-theory on all toric hypersurface fibrations and its Higgs branches, JHEP 01 (2015) 142 [arXiv: 1408.4808] [INSPIRE].

[31] Y.-C. Huang and W. Taylor, Mirror symmetry and elliptic Calabi-Yau manifolds, JHEP 04 (2019) 083 [arXiv: 1811.04947] [INSPIRE].

[32] http://ctp.lns.mit.edu/wati/data.html

[33] P. Candelas and A. Font, Duality between the webs of heterotic and type-II vacua, Nucl. Phys. B 511 (1998) 295 [hep-th/9603170] [INSPIRE].

[34] P. Berglund, Y.C. Huang, W. Taylor and Y.N. Wang, to appear.

[35] J. Halverson, C. Long and W. Taylor, to appear.

[36] P. Candelas et al., Codimension three bundle singularities in F-theory, JHEP 06 (2002) 014 [hep-th/0009228] [INSPIRE].

[37] C. Lawrie and S. Schäfer-Nameki, The Tate form on steroids: resolution and higher codimension fibers, JHEP 04 (2013) 061 [arXiv:1212.2949] [INSPIRE].

[38] J. Borchmann, C. Mayrhofer, E. Palti and T. Weigand, Elliptic fibrations for $\mathrm{SU}(5) \times \mathrm{U}(1) \times \mathrm{U}(1)$ F-theory vacua, Phys. Rev. D 88 (2013) 046005 [arXiv: 1303.5054] [INSPIRE].

[39] M. Cvetič, A. Grassi, D. Klevers and H. Piragua, Chiral four-dimensional F-theory compactifications with $\mathrm{SU}(5)$ and multiple $\mathrm{U}(1)$-factors, JHEP 04 (2014) 010 [arXiv: 1306.3987] [INSPIRE].

[40] M. Dierigl, P.-K. Oehlmann and F. Ruehle, Global tensor-matter transitions in F-theory, Fortsch. Phys. 66 (2018) 1800037 [arXiv:1804.07386] [INSPIRE]. 
[41] I. Achmed-Zade, I. García-Etxebarria and C. Mayrhofer, A note on non-flat points in the $\mathrm{SU}(5) \times \mathrm{U}(1)_{P Q}$ F-theory model, JHEP 05 (2019) 013 [arXiv: 1806. 05612] [INSPIRE].

[42] J. Tian and Y.-N. Wang, E-string spectrum and typical F-theory geometry, arXiv: 1811.02837 [INSPIRE].

[43] F. Apruzzi, L. Lin and C. Mayrhofer, Phases of 5d SCFTs from M-/F-theory on Non-Flat Fibrations, JHEP 05 (2019) 187 [arXiv:1811.12400] [INSPIRE].

[44] F. Apruzzi et al., Fibers add flavor. Part I: classification of $5 d$ SCFTs, flavor symmetries and BPS states, JHEP 11 (2019) 068 [arXiv:1907.05404] [INSPIRE].

[45] P. Berglund and T. Hubsch, A generalized construction of Calabi-Yau models and mirror symmetry, SciPost Phys. 4 (2018) 009 [arXiv: 1611.10300] [INSPIRE].

[46] D.R. Morrison and W. Taylor, Classifying bases for $6 D$ F-theory models, Central Eur. J. Phys. 10 (2012) 1072 [arXiv:1201.1943] [InSPIRE].

[47] A. Klemm, B. Lian, S.S. Roan and S.-T. Yau, Calabi-Yau fourfolds for M-theory and F-theory compactifications, Nucl. Phys. B 518 (1998) 515 [hep-th/9701023] [INSPIRE].

[48] W. Taylor and Y.-N. Wang, A Monte Carlo exploration of threefold base geometries for $4 d$ F-theory vacua, JHEP 01 (2016) 137 [arXiv:1510.04978] [INSPIRE].

[49] M. Demirtas, C. Long, L. McAllister and M. Stillman, The Kreuzer-Skarke axiverse, arXiv: 1808.01282 [INSPIRE].

[50] R. Friedman, J. Morgan and E. Witten, Vector bundles and F-theory, Commun. Math. Phys. 187 (1997) 679 [hep-th/9701162] [INSPIRE].

[51] R. Davies, The expanding zoo of Calabi-Yau threefolds, Adv. High Energy Phys. 2011 (2011) 901898 [arXiv: 1103.3156] [INSPIRE].

[52] V. Braun, T.W. Grimm and J. Keitel, Complete intersection fibers in F-theory, JHEP 03 (2015) 125 [arXiv:1411.2615] [INSPIRE].

[53] M.-x. Huang, S. Katz and A. Klemm, Topological string on elliptic CY 3-folds and the ring of Jacobi forms, JHEP 10 (2015) 125 [arXiv:1501.04891] [INSPIRE].

[54] J. Gray, A.S. Haupt and A. Lukas, Topological invariants and fibration structure of complete intersection Calabi-Yau four-folds, JHEP 09 (2014) 093 [arXiv: 1405. 2073] [INSPIRE].

[55] F. Schöller and H. Skarke, All weight systems for Calabi-Yau fourfolds from reflexive polyhedra, Commun. Math. Phys. 372 (2019) 657 [arXiv: 1808.02422] [INSPIRE].

[56] J. Halverson, C. Long and B. Sung, Algorithmic universality in F-theory compactifications, Phys. Rev. D 96 (2017) 126006 [arXiv:1706.02299] [INSPIRE].

[57] W. Taylor and Y.-N. Wang, Scanning the skeleton of the 4D F-theory landscape, JHEP 01 (2018) 111 [arXiv:1710.11235] [INSPIRE]. 